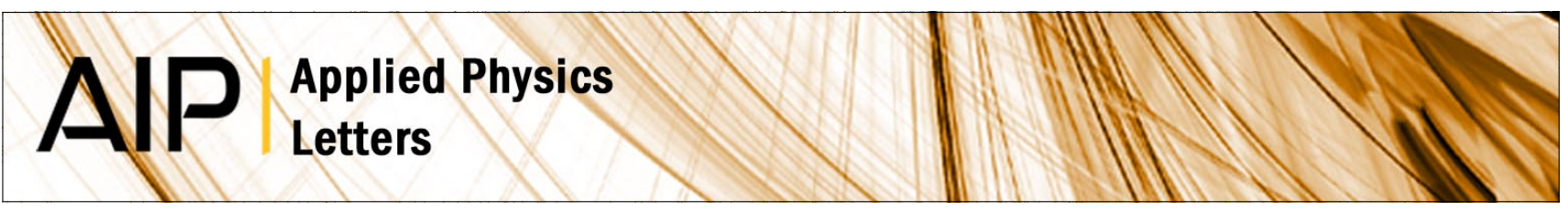

\title{
Band gap energy determination by photoacoustic spectroscopy under continuous light excitation
}

N. G. Astrath, F. Sato, F. Pedrochi, A. N. Medina, A. C. Bento et al.

Citation: Appl. Phys. Lett. 89, 231926 (2006); doi: 10.1063/1.2402239

View online: http://dx.doi.org/10.1063/1.2402239

View Table of Contents: http://apl.aip.org/resource/1/APPLAB/v89/i23

Published by the American Institute of Physics.

Additional information on Appl. Phys. Lett.

Journal Homepage: http://apl.aip.org/

Journal Information: http://apl.aip.org/about/about_the_journal

Top downloads: http://apl.aip.org/features/most_downloaded

Information for Authors: http://apl.aip.org/authors

\section{ADVERTISEMENT}

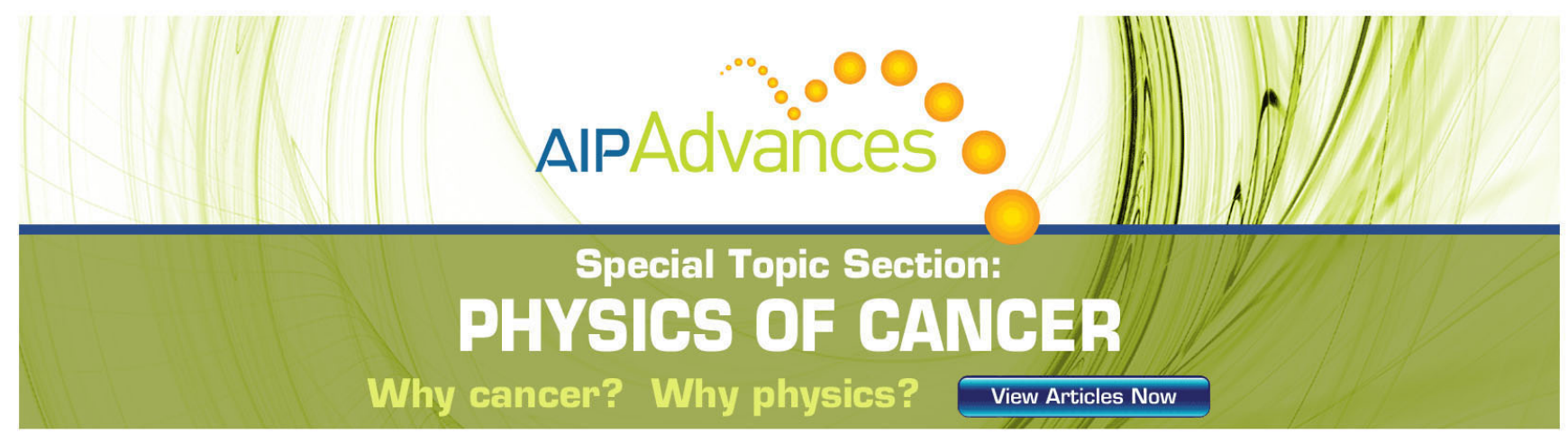




\title{
Band gap energy determination by photoacoustic spectroscopy under continuous light excitation
}

\author{
N. G. C. Astrath, ${ }^{\text {a) }}$ F. Sato, F. Pedrochi, A. N. Medina, A. C. Bento, and M. L. Baesso \\ Departamento de Física, Universidade Estadual de Maringá, 87020-900 Maringá, PR, Brazil \\ C. Persson \\ Department of Materials Science and Engineering, Royal Institute of Technology, SE-100 44 Stockholm, \\ Sweden

\begin{abstract}
A. Ferreira da Silva
Instituto de Física, Universidade Federal da Bahia, Campus Universitário de Ondina, 40210-340 Salvador, BA, Brazil
\end{abstract}

(Received 1 September 2006; accepted 19 October 2006; published online 7 December 2006)

\begin{abstract}
In this work the authors used the photoacoustic spectroscopy under continuous light excitation to determine the optical band gap of semiconductors. The experiments were performed in lead iodide $\mathrm{PbI}_{2}$ and hexagonal silicon carbide $4 H-\mathrm{SiC}$ samples. The nonradiative relaxation processes are discussed in terms of the generated signal. A mechanism to describe the signal increase/decrease under the continuous excitation is presented. The results showed that the method was useful to locate the band gap directly from the optical absorption spectra. (C) 2006 American Institute of Physics. [DOI: 10.1063/1.2402239]
\end{abstract}

Photoacoustic Spectroscopy (PAS) has been extensively used to determine the optical properties of semiconductor materials via energy transfer processes that result in heat generation. ${ }^{1-6}$ PAS and most of the photothermal methods can provide information regarding the carrier transport properties if the absorbed energy, $h \nu$, is higher than the band gap energy $\left(E_{g}\right){ }^{1-9}$ In this case, an excess of photoinjected carriers with energy $h \nu-E_{g}$ in the conduction band is created, followed by electron-phonon collision deexcitation that occurs in the picoseconds scale. Then, as the carriers give off the excess of energy to the lattice, they diffuse through the sample and reestablish equilibrium by recombination with the holes in the valence band.

One particular characteristic of the PAS is the fact that the mechanisms responsible for the generation of the photoacoustic signal (PS) can be significantly modified depending on whether the measurements are performed with photon energy above or below the band gap. Another important aspect of the technique is that the effective thickness of the sample that contributes to the PS is defined by the thermal diffusion length $(\mu)$, the light penetration depth $\left(1_{\beta}(\lambda)=1 / \beta(\lambda)\right)$, with $\beta$ as the optical absorption coefficient and $\lambda$ the light wavelength, and the nonradiative relaxation time $(\tau)$, which is a time constant directly related to the phase of the PS. Then, the evaluation of the mentioned parameters allows the use of the technique to perform depth profile analysis and energy transfer processes. In the case of semiconductors, bulk and surface absorptions, below and above the band gap, respectively, can be discriminated with this method. ${ }^{7}$

One of the most important parameter for the electronic and optoelectronic areas ${ }^{8-12}$ is the energy band gap of semiconductors. Usually $E_{g}$ value is adopted as the absorption edge obtained from a linear fitting in the plot of the square of the absorption coefficient ${ }^{13}$ versus the photon energy for direct band gap, or the plot of the square root of the product between the absorption coefficient and the phonon energy

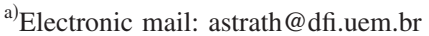

versus the photon energy ${ }^{14}$ for indirect band gap. However, this procedure is not always precise, since the absolute value of $\beta(\lambda)$ in the region where the band gap occurs is not simple to obtain, once it depends on the sample preparation conditions, purity of the used raw materials, and surface optical finishing. ${ }^{1,14}$ Several techniques have been applied to obtain the optical absorption spectra that can be used for the band gap determination, including PAS and more recently photoreflectance..$^{7-9,15}$ The use of conventional PAS is limited when the nonradiative recombination in the band edge is minimized, while with the photoreflectance techniques usually the fluorescence cannot be separated from the probe light. Therefore, the search for other methods for the precise determination of the semiconductors band gap is desirable.

In this work we analyze the behavior of the PS under continuous laser excitation in two semiconductor samples, the lead iodide $\mathrm{PbI}_{2}$ and the hexagonal silicon carbide $4 H$-SiC, using an experimental procedure similar to that previously applied to study the oxygen evolution in plant leaves. ${ }^{16}$ The influence of the continuous excitation in the mechanisms responsible for the generation of the PS is discussed and adopted to determine the energy of the band gaps directly from the experimental optical absorption spectra.

The testing samples were a bulk single crystal $\mathrm{PbI}_{2}$ and a thin film $4 \mathrm{H}$-SiC crystal. The $\mathrm{PbI}_{2}$ crystal was grown by the Bridgman method with the $c$ axis oriented perpendicularly to the growth axis. ${ }^{17,18}$ The $4 \mathrm{H}$-SiC sample with doping around $7 \times 10^{18} \mathrm{~cm}^{-3}$ was grown by hot wall chemical vapor deposition. ${ }^{19}$

Photoacoustic spectra were measured between 1.5 and $6.2 \mathrm{eV}$ and normalized with fine carbon black. The experimental setup was a photoacoustic spectrometer with the samples placed in a conventional PAS cell. The light source was a $1000 \mathrm{~W}$ Xe-arc lamp and was modulated at a frequency of $20 \mathrm{~Hz}$. The PS signal was detected by a Bruel and Kjaer microphone and processed by a 5210 PAR lock-in amplifier. $\mathrm{An}^{+}{ }^{+}$ion laser tuned at 2.72 and $2.41 \mathrm{eV}$ was used as continuous light source, while at 1.96 and $2.33 \mathrm{eV}$ a $\mathrm{He}-\mathrm{Ne}$ and a neodymium doped yttrium aluminum garnet 


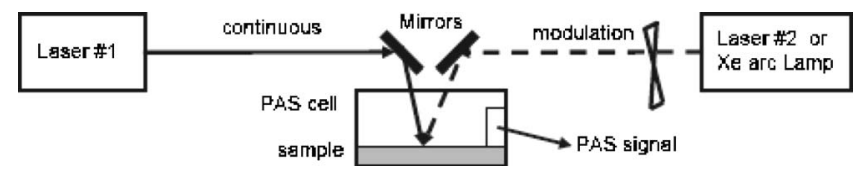

FIG. 1. Sketch used in the photoacoustic detection under continuous light excitation.

lasers were used, respectively. First, the photoacoustic spectra were measured while the samples were excited only by the modulated light. Then, the spectra were obtained with simultaneous excitation by the continuous laser light, as can be seen in Fig. 1. In order to better understand the influence of the continuous light over the PS, an additional experiment was performed as follows: the wavelengths of the modulated and continuous light are maintained fixed and the PS is measured as a function of time. In this way, by monitoring the PS when the sample is irradiated with the continuous light, it is possible to determine whether there is an increase or a decrease of the signal.

Figure 2 shows the spectra and the phase for lead iodide with or without excitation by continuous laser light as a function of photon energy. The spectrum of the nonirradiated sample shows an onset of the optical absorption band around $2.3 \mathrm{eV}$ and a broad band around $4.5 \mathrm{eV}$. The latter is in agreement with previous results for band gap determination using the dielectric function ${ }^{20}$ and the reflectivity. ${ }^{21}$ This peak at $4.5 \mathrm{eV}$ is present in the calculated imaginary part of dielectric function $\varepsilon_{2}^{\perp}, \varepsilon_{2}^{\|}$, and $\varepsilon_{2}^{\text {tot }}$ (Ref. 20), which is in good agreement with the calculated spectra, being originated from $\mathrm{Pb}-s$ to $\mathrm{Pb}-p$ interband transition. ${ }^{20}$ In addition, our calculations using the first-principles full-potential within the local density approximation (LDA) plus an estimated quasiparticle (QP) correction (as described in Ref. 22) yield a direct fundamental band gap energy for this material as $E_{g}$ (LDA $+\mathrm{QP})=2.52 \mathrm{eV}$. In Fig. 2(b), the decrease in the phase values as the band gap approaches is expected since $1_{\beta}$ decreases due to the higher values of the optical absorption coefficient. Then, the PS is generated in a lower depth in the sample reducing both the nonradiative relaxation time and the phase of the signal. The increase in the phase values above the band gap suggests the occurrence of changes in the optical absorption or in the thermal relaxation mechanisms, as the nonradiative relaxation time appears to increase. ${ }^{23}$

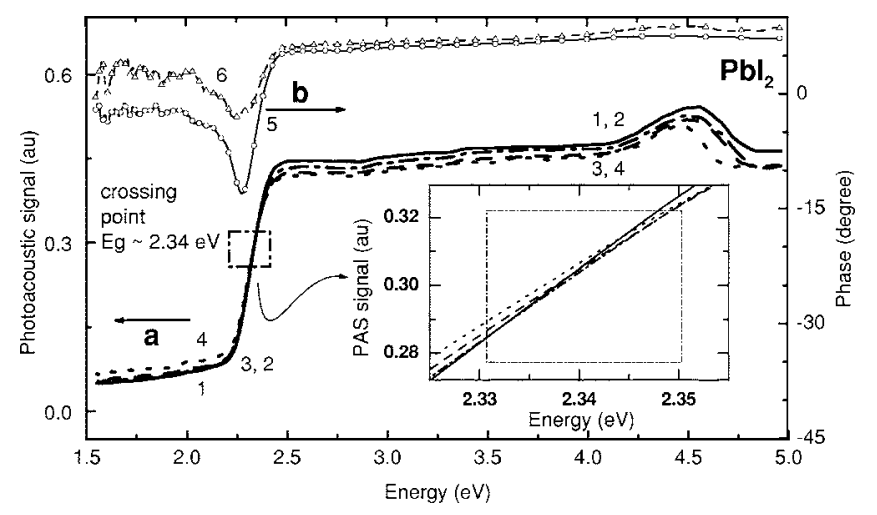

FIG. 2. PS (a) and phase (b) of $\mathrm{PbI}_{2}$ against photon energy. The crossing point with and without continuous excitation (shown in the inset) determines $E_{g}=2.34 \pm 0.01 \mathrm{eV}$. Curves (a) are a1 for unirradiated, a2 for continuous $40 \mathrm{~mW}$ at $2.72 \mathrm{eV}$, a3 for continuous $40 \mathrm{~mW}$ at $2.41 \mathrm{eV}$, and a4 continuous $15 \mathrm{~mW}$ at $1.96 \mathrm{eV}$. Curves (b) are b5, phase of a1 and b6, phase of a2.

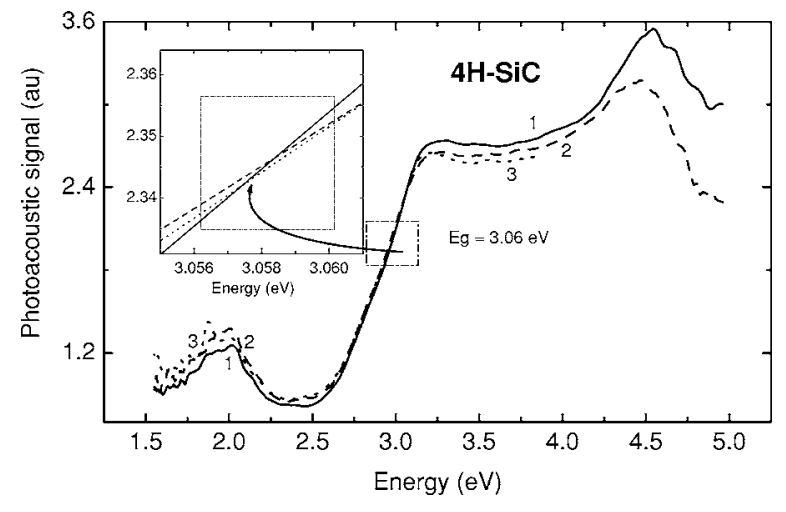

FIG. 3. PS of $n$-type doped $4 \mathrm{H}-\mathrm{SiC}$ against photon energy. The indirect band gap $E_{g}=3.06 \pm 0.01 \mathrm{eV}$ is obtained as in Fig. 2(a) (see inset). The peak at $\sim 4.5 \mathrm{eV}$ originates from direct $M_{v} \rightarrow M_{c}$ transition. Curves are 1 unirradiated, and for continuous $70 \mathrm{~mW}$ being 2 at $2.72 \mathrm{eV}$, and 3 at $2.41 \mathrm{eV}$.

As mentioned before, the determination of the band gap energy is usually a challenge task. For example, for the $\mathrm{PbI}_{2}$ sample, by assuming the band gap value as the photon energy at the absorption edge, $E_{g}=2.35 \pm 0.05 \mathrm{eV}$ can be identified as the fundamental band gap energy. ${ }^{20}$ However, if the optical band gap is evaluated from the plot of the square of the absorption coefficient $\left(\beta^{2}\right)$ versus the photon energy, ${ }^{24}$ $E_{g}=2.28 \pm 0.01 \mathrm{eV}$. In this manner, the correct value of the band gap energy is difficult to establish due to the uncertainties in the determination of the absolute value of $\beta(\lambda)$ in the band gap edge. Thus, by excitation with continuous light, as shown in Fig. 2, the generated PS changes for energies higher and lower than the one of the band gap, but they can be considered the same in the region where the nonirradiated and irradiated continuous light spectra cross. The crossing of the spectra, as shown in details in the inset of Fig. 2, can be assumed as the value of the band gap energy, i.e., $E_{g}$ $=2.34 \pm 0.01 \mathrm{eV}$. This procedure appears to be useful to locate the band gap energy of the sample. It can also be seen in Fig. 2(b) that there is an increase in the phase of the PS under continuous excitation, occurred for all used wavelengths. We will go back to this observation later on in the discussion of the mechanism responsible for the change in the behavior of the PS under continuous excitation as compared to the regular spectrum. In Fig. 3, the same procedure used for $\mathrm{PbI}_{2}$ is applied to analyze the $4 H-\mathrm{SiC}$, a thin film sample. In this case, the energy from the continuous laser is in the band gap region. This fact and the high reflectivity of this sample, which results in very low optical absorption coefficient as compared to $\mathrm{PbI}_{2}$, demanded special care to avoid the contribution of the back scattering. This was done by subtracting the background obtained by measuring the spectrum with the Xe lamp off and the continuous laser light on. The final spectrum shown in Fig. 3 was obtained by averaging three spectra determined in the same condition. By adopting again the crossing region of the spectra for the nonirradiated and irradiated samples as the band gap energy, the achieved value for $E_{g}$ is $(3.06 \pm 0.01) \mathrm{eV}$. This is the fundamental band gap of this material, originated from the phonon assisted optical transition $\Gamma_{v} \rightarrow M_{c}$. It can be noted also that two other semiconductor absorption bands are present at $2.00 \mathrm{eV}$ and around $4.50 \mathrm{eV}$. The $\sim 4.5 \mathrm{eV}$ absorption is identified from the first-principles calculations ${ }^{22,25}$ as the $M$-point optical direct transition $M_{v} \rightarrow M_{c}$. The absorption band around $2.0 \mathrm{eV}$, which is about $1.1 \mathrm{eV}$ below the conduction band edge, is 


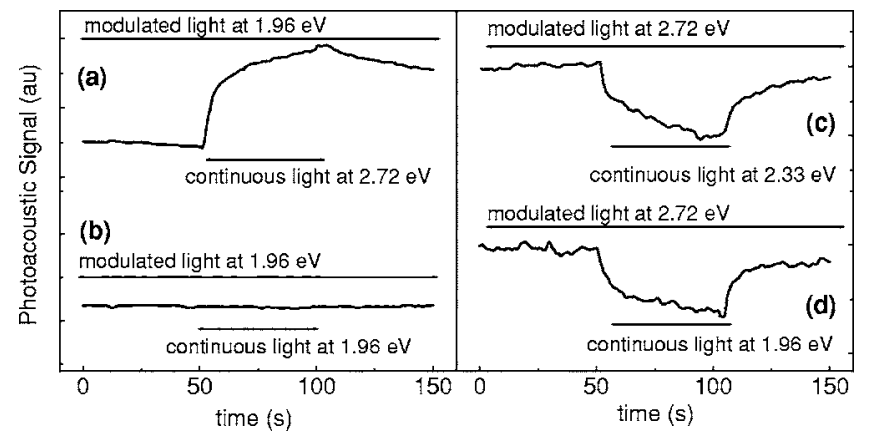

FIG. 4. PS time response for $\mathrm{PbI}_{2}$. Continuous light impinged the sample from 50 to $100 \mathrm{~s}$. Left: the modulated light is at $1.96 \mathrm{eV}\left(h \nu<E_{g}\right)$. Curve (a): continuous light above bandgap, and curve (b) continuous light at $1.96 \mathrm{eV}\left(h \nu<E_{g}\right)$. Right: in curves (c) and (d) modulated light are for $h \nu$ $>E_{g}$. Note PS reduction with continuous $h \nu<E_{g}$, curve (d).

identified as $\mathrm{Cr}$ or $\mathrm{V}$ levels in $4 \mathrm{H}-\mathrm{SiC}$ as pointed out by Achtziger and Witthuhn. ${ }^{26}$ As noted for the $\mathrm{PbI}_{2}$ sample, the phase of the PS for $4 \mathrm{H}-\mathrm{SiC}$ also increased as the continuous light impinged the sample (not shown).

In order to understand the decreasing and the increasing of the generated PS with the continuous light excitation, a further experiment was performed by monitoring the PS as a function of time, as shown in Fig. 4 for $\mathrm{PbI}_{2}$. The same effects observed in the spectra are shown in the time response regime of the PS when the modulated and continuous wavelengths are maintained fixed. The increase of the PS in curve (a) occurs when the modulated light is fixed at $1.96 \mathrm{eV}$ and the continuous light fixed at $2.72 \mathrm{eV}$. In the first $50 \mathrm{~s}$, when only the modulated light $(1.96 \mathrm{eV})$ is incident on the sample, the PS is low since the absorbed energy is not sufficient for the electrons to reach the conduction band. Then, the PS is generated by the absorption that occurs in the conduction band via thermally assisted interband transition, with the absorption of energy $k_{B} T$ over the Fermi level allowing some low free carrier concentration, with $n_{e} \sim 0$, but $>0$. It is important to mention that the higher values of the phase of the PS [Fig. 2(b)] under continuous excitation would imply in a reduction in the PS as a consequence of a higher nonradiative relaxation time. However, as mentioned above, a significant increase in the signal was observed between 50 and $100 \mathrm{~s}$ in Fig. 4(a). Our hypothesis to explain this result is that, when continuous light with energy higher than the band gap is incident, the excited electrons are promoted to the conduction band. Then the continuous light induced free carriers absorb the modulated light resulting in an intraband transitions. As a consequence of the higher probability of the modulated light to be absorbed by the excess of free carriers, a higher PS is generated. This mechanism may dominate the signal generation, despite the observed increase in the phase values. This interpretation is also consistent with the results of Fig. 4(b), since although the continuous light at $1.96 \mathrm{eV}$ (lower than the band gap) can be absorbed by the free carriers, it is not chopped and then can not contribute to the PS.

The decrease of the PS occurs when the modulated light is fixed at $2.72 \mathrm{eV}$ and the continuous light at 2.33 and $1.96 \mathrm{eV}$, shown in Fig. 4 as curves (c) and (d). In the first $50 \mathrm{~s}$, the modulated light is sufficient for the excitement of the electrons to the conduction band and from there they decay nonradiatively to the valence band generating the PS. When the continuous light is incident, some conduction electrons (free carriers) may absorb energy at the conduction band inducing an intraband transition. In this process, there is an increase in the nonradiative relaxation time [this is confirmed by the higher phase values shown in Fig. 2(b)] and since the PS is inversely proportional to the time of the recombination processes, the generated signal decreases as shown in the time interval between 50 and $100 \mathrm{~s}$ in Fig. 4, curves (c) and (d). As the continuous light is off, after $100 \mathrm{~s}$, the PS recovers to its original value.

To sum up, in the present work we used the PAS under continuous laser excitation to propose a tool to determine the optical band gap energy of semiconductors. The band gap energy found for $\mathrm{PbI}_{2}$ and $4 H-\mathrm{SiC}$ were $2.34 \pm 0.01 \mathrm{eV}$ and $3.06 \pm 0.01 \mathrm{eV}$, respectively. The relaxation processes in the structure of the semiconductor's energy bands is discussed in terms of the generated PS. The experiments are simple to perform, widening the number of available methods for the study of the energy transfer processes and band gap determination in semiconductors.

The authors acknowledge CAPES, CNPq, REMAN/ CNPq, Swedish Research Council (VR) and the Agency STINT, and Fundação Araucária for the financial support of this work.

${ }^{1}$ L. Eaves, H. Vargas, and P. J. Williams, Appl. Phys. Lett. 38, 768 (1981).

${ }^{2}$ A. Mandelis, Photoacoustic and Thermal Wave Phenomena in Semiconductors (North-Holland, New York, 1987), Vol. 1, p. 397.

${ }^{3}$ O. Zelaya-Angel, J. J. Alvarado-Gil, R. Lozada-Morales, H. Vargas, and A. Ferreira da Silva, Appl. Phys. Lett. 64, 291 (1994).

${ }^{4}$ T. Kawahara, Y. Ohbuchi, J. Morimoto, H. Goto, and T. Ido, Mater. Lett. 50, 347 (2001).

${ }^{5}$ H. Vargas and L. C. M. Miranda, Phys. Rep. 161, 43 (1988).

${ }^{6}$ A. Pinto Neto, H. Vargas, N. F. Leite, and L. C. M. Miranda, Phys. Rev. B 41, 9971 (1990)

${ }^{7}$ H. Yamamoto, I. Suemune, and M. Yamanishi, J. Appl. Phys. 60, 2621 (1986).

${ }^{8}$ L. P. Avakyants, P. Y.Bokov, T. P. Kolmakova, and A. V. Chervyakov, Semiconductors 38, 1384 (2004).

${ }^{9}$ A. E. Sotnikov, M. A. Chernikov, and O. A. Ryabushkin, Instrum. Exp. Tech. 47, 656 (2004).

${ }^{10}$ K. F. Berggren and B. E. Sernelius, Phys. Rev. B 24, 1971 (1981).

${ }^{11}$ W. P. Dumke, Appl. Phys. Lett. 42, 196 (1983).

${ }^{12}$ K. E. Newman, A. Lastras-Martinez, B. Kramer, S. A. Barnett, M. A. Ray, J. D. Dow, J. E. Greene, and P. M. Raccach, Phys. Rev. Lett. 50, 1466 (1983).

${ }^{13}$ A. Nayak, G. L. Bhalla, and G. C. Trigunayat, Mater. Res. Bull. 35, 2263 (2000).

${ }^{14}$ D. L. Wood and J. Tauc, Phys. Rev. B 5, 3144 (1972).

${ }^{15}$ L. R. de Freitas, E. C. da Silva, A. M. Mansanares, G. Tessier, and D. Fournier, J. Appl. Phys. 98, 063508 (2005).

${ }^{16}$ R. C. Mesquita, A. M. Mansanares, E. C. da Silva, P. R. Barja, L. C. M. Miranda, and H. Vargas, Instrum. Sci. Technol. 34, 33 (2006).

${ }^{17}$ T. S. Silva, A. S. Alves, I. Pepe, H. Tsuzuki, O. Nakamura, M. M. F. d'Aguiar Neto, A. Ferreira da Silva, N. Veissid, and C. Y. An, J. Appl. Phys. 83, 6193 (1998).

${ }^{18}$ A. Ferreira da Silva, N. Veissid, C. Y. An, I. Pepe, N. Barros de Oliveira, and A. V. Batista da Silva, Appl. Phys. Lett. 69, 1930 (1996).

${ }^{19}$ Q. Wahab, A. Ellison, A. Henry, E. Janzén, C. Hallin, J. Di Persio, and R. Martinez, Appl. Phys. Lett. 76, 2725 (2000).

${ }^{20}$ R. Ahuja, H. Arwin, A. Ferreira da Silva, C. Persson, J. M. OsorioGuillén, J. Souza de Almeida, C. Moyses Araújo, E. Veje, N. Veissid, C. Y.

Na, I. Pepe, and B. Johansson, J. Appl. Phys. 92, 7219 (2002).

${ }^{21}$ I. Ch. Schlüter and M. Schlüter, Phys. Rev. B 9, 1652 (1974).

${ }^{22}$ C. Persson and A. Ferreira da Silva, in Optoelectronic Devices: IIINitrides, edited by M. Razeghi and M. Henini (Elsevier, Oxford, 2004), Vol. 1, p. 479.

${ }^{23}$ D. P. Almond and P. M. Patel, Photothermal Science and Techniques (Chapman and Hall, London, 1996), Vol. 10, pp. 130-133.

${ }^{24}$ R. H. Bube, Electronic Properties of Crystalline Solids (Academic, New York, 1974), Vol. 1, pp. 438-439.

${ }^{25}$ C. Persson and U. Lindefelt, J. Appl. Phys. 86, 5036 (1999).

${ }^{26}$ N. Achtziger and W. Witthuhn, Appl. Phys. Lett. 71, 110 (1997). 